\title{
All-Aqueous Liquid Crystal Nanocellulose Emulsions with Permeable Interfacial Assembly
}

Long Bai, ${ }^{\#}$ Siqi Huan, ${ }^{\#}$ Bin Zhao, Ya Zhu, Jordi Esquena, Feng Chen, Guang Gao, Eyal Zussman, Guang Chu,* and Orlando J. Rojas*

Cite This: ACS Nano 2020, 14, 13380-13390

Read Online

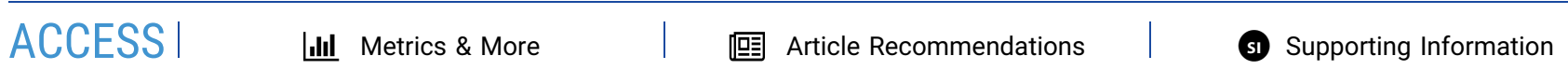

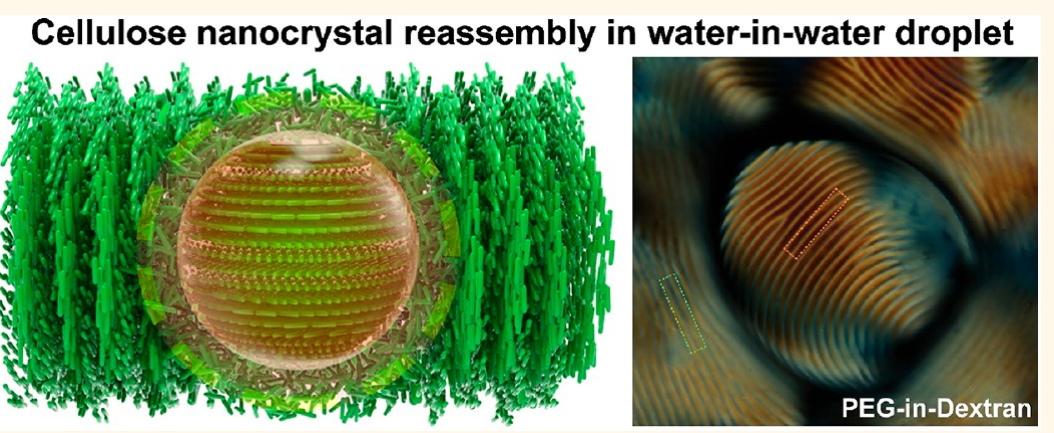

ABSTRACT: We report on the formation of water-in-water liquid crystal emulsions with permeable colloidal assemblies. Rodlike cellulose nanocrystals (CNC) spontaneously self-assemble into a helical arrangement with the coexistence of nonionic, hydrophilic polyethylene glycol (PEG) and dextran, whereas the two polymer solutions are thermodynamically incompatible. Stable water-in-water emulsions are easily prepared by mixing the respective $\mathrm{CNC}$ /polymer solutions, showing micrometric CNC/PEG dispersed droplets and a continuous CNC/dextran phase. With time, the resulting emulsion demixes into an upper, droplet-lean isotropic phase and a bottom, droplet-rich cholesteric phase. Owing to the osmotic pressure gradient between PEG and dextran phases, target transfer of cellulose nanoparticles occurs across the water/water interface to reassemble into a liquid crystal-in-liquid crystal emulsion with global cholesteric organization. The observed structural, optical, and temporal evolution confirm that the colloidal particles in the two immiscible phases experience short-range interactions and form long-range assemblies across the interface.

KEYWORDS: liquid crystal, water-in-water emulsion, cellulose nanocrystals, osmotic pressure, permeable self-assembly

$\mathrm{E}$ mulsions are two-phase systems that typically consist of two immiscible solvents, with one liquid dispersed into the other, forming kinetically stable droplets. ${ }^{1}$ They form water-in-oil $(\mathrm{W} / \mathrm{O})$ or oil-in-water $(\mathrm{O} / \mathrm{W})$ emulsions that can be found in dairy formulations, pharmaceuticals, and household products. ${ }^{2,3}$ A less common type of liquid/liquid biphasic system corresponds to water-in-water (W/W) emulsions with droplets of one aqueous phase suspended in the other. Phase segregation in two liquids occurs because of the presence of different hydrophilic molecules that are entirely water-soluble but mutually incompatible., ${ }^{4,5}$ Compared with the water/oil interfaces, the water/water interfaces present in $\mathrm{W} / \mathrm{W}$ emulsions display ultralow interfacial tension (around $0.01-100 \mu \mathrm{N} / \mathrm{m},{ }^{6}$ depending on the proximity to the critical point) and large interfacial thickness (on the order of several nanometers) along with small variations of macroscopic properties across the interface. Hence, they form a "fuzzy boundary" that is highly permeable to water, ions, and small molecules. ${ }^{6-8}$ The interplay between the two equilibrium phases leads to small but measurable concentration and chemical potential gradients across the interface, ${ }^{9-11}$ such as those found in biological W/W systems (cells, vesicles, and liposomes).

Received: June 24, 2020

Accepted: September 18, 2020

Published: September 18, 2020 

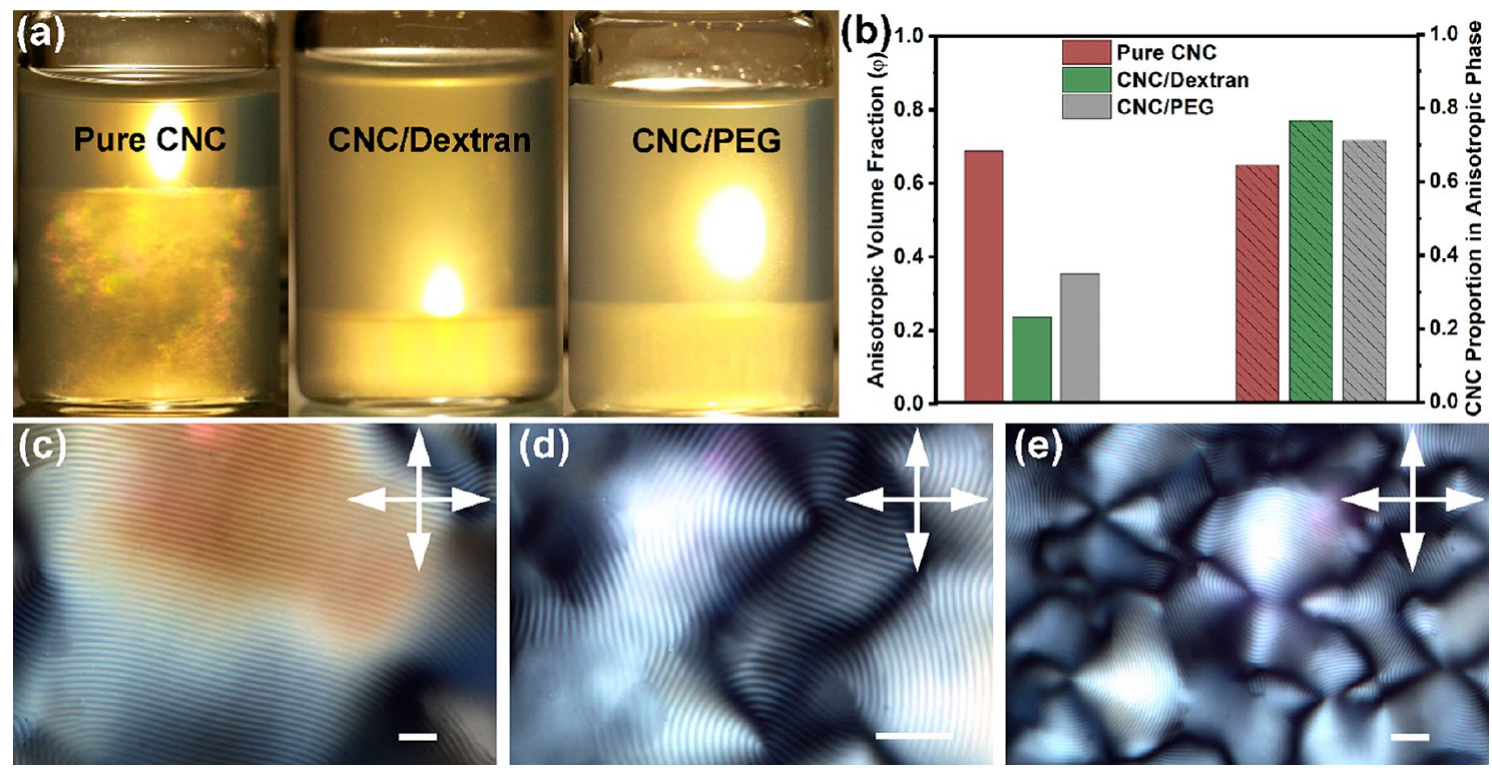

Figure 1. Phase separation of $\mathrm{CNC}$ /polymer suspension with cholesteric organization. (a) Photographs of the CNC binary suspension with the addition of dextran and PEG, respectively. The CNC concentration in the suspensions was 5 wt \%. (b) Calculated volume fraction and proportion of $\mathrm{CNC}$ in the anisotropic phase for suspensions with and without the addition of polymer. POM images of the cholesteric phase for (c) pure CNC, (d) CNC/dextran, and (e) CNC/PEG suspensions, showing periodic fingerprint textures. Scale bar: $50 \mu \mathrm{m}$.

Emulsions with at least one phase composed of an anisotropic fluid are termed as "liquid crystal (LC) emulsion". ${ }^{12-14}$ LC emulsions exert striking impacts in structures for both $\mathrm{W} / \mathrm{O}$ and $\mathrm{O} / \mathrm{W}$ systems, and various kinds of complex assemblies have been observed due to the anisotropic elasticity, interfacial interactions, elastic distortions, and topological defects, etc. ${ }^{15-19}$ Some anisotropic colloidal particles (e.g., $\mathrm{V}_{2} \mathrm{O}_{5}$, graphene oxide, virus, and amyloid fibrils) can form aqueous lyotropic LCs at high concentrations. ${ }^{20-24}$ During the LC formation process, microdomains of the anisotropic droplet spontaneously nucleate from the isotropic suspension. ${ }^{25,26}$ The obtained LC droplets, denoted as tactoid, result from a transition state between isotropic and macroscopic liquid crystalline phases, which can be classified as a special type of W/W LC emulsion. Yet, it is metastable, and the small tactoids spontaneously merge into larger ones, finally transforming into a macroscopic LC phase. ${ }^{27}$ In order to prepare a stable $\mathrm{W} / \mathrm{W}$ emulsion, hydrophilic polymers are added to generate repulsive forces between the two aqueous phases. $^{28}$ Therefore, a key point that arises is the need for suitable polymers that can be dispersed in the LC system, allowing the LCs to coexist without disrupting the structure of the emulsion and LC ordering.

This brings consideration of cellulose nanocrystals (CNC), which are chiral, charged, and rodlike nanoparticles that form stable colloidal suspensions in water. ${ }^{29,30}$ At low CNC concentrations, the suspension is isotropic, becoming anisotropic above a critical concentration, as nanocrystals selfassemble into an ordered helical arrangement, in agreement with Onsager theory for rigid rodlike particles. ${ }^{31,32}$ Unlike molecular-based lyotropic and thermotropic LCs, the CNC cholesteric phase is extremely stable, even in the presence of polymers, nanoparticles, surfactants, and inorganic precursors, ${ }^{33-39}$ providing a robust matrix for designing functional materials. CNC-based LCs have received considerable attention for their chiral, self-assembly, and photonic properties as well as templating capabilities. ${ }^{40-45}$ In contrast, to the best of our knowledge, no report exists on the use of CNC in LC emulsions with hierarchical $\mathrm{W} / \mathrm{W}$ organization, particularly, in fully aqueous LC-in-LC emulsions. Whereas several studies have demonstrated $\mathrm{CNC}$ as an effective stabilizer of $\mathrm{O} /$ $\mathrm{W}$ emulsions, ${ }^{46}$ so far only two reports have considered $\mathrm{W} / \mathrm{W}$ systems. $^{47,48}$ Thus, the case of LC emulsions based on cellulose-derived nanomaterials remains as an uncharted topic. Fundamentally, CNC-based W/W LC emulsions are quite attractive and offer excellent opportunities in the development of all-aqueous, advanced multiphase systems.

Herein, we develop a series of W/W LC emulsions with global cholesteric organization. Specifically, we describe the use of colloidal suspensions of $\mathrm{CNC} /$ dextran as a continuous phase to suspend immiscible droplets of $\mathrm{CNC} /$ polyethylene glycol (PEG). We show that CNC particles can sustain the cholesteric ordering with the addition of hydrophilic dextran and PEG at high concentrations. When the two $\mathrm{CNC} /$ polymer solutions are mixed, a stable $\mathrm{W} / \mathrm{W}$ emulsion is obtained, namely, CNC/PEG droplets dispersed into a continuous $\mathrm{CNC} /$ dextran phase. During equilibrium, the cellulose nanoparticles in the PEG phase diffuse into the continuous dextran phase due to the osmotic pressure gradient and reassemble into a global cholesteric arrangement across the PEG/dextran interface. Here, the structure, optical texture, and temporal evolution of a W/W LC emulsion are studied in detail, showing a close relationship with the polymer-induced osmotic pressure in each phase. Our approach provides a generic framework to formulate and prepare W/W LC emulsions with predefined organization, which can be extended to other anisotropic colloidal particles.

\section{RESULTS AND DISCUSSION}

Cholesteric Assembly in Immiscible Phases. The CNC was prepared by controlled sulfuric acid hydrolysis of wood fibers that yielded polydispersed nanoparticles with rodlike morphologies (300-400 nm in length and 10-15 nm in diameter, Figure S1) with highly electronegative charges $(\zeta$ - 
(a)

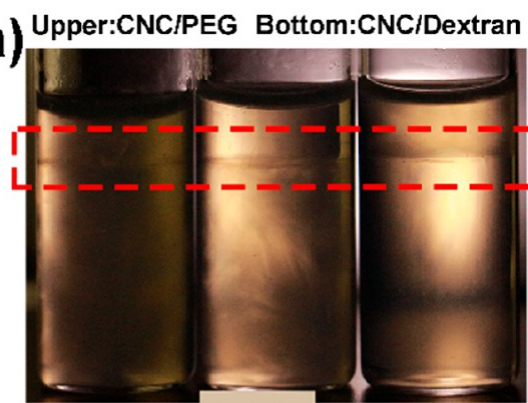

Emulsification
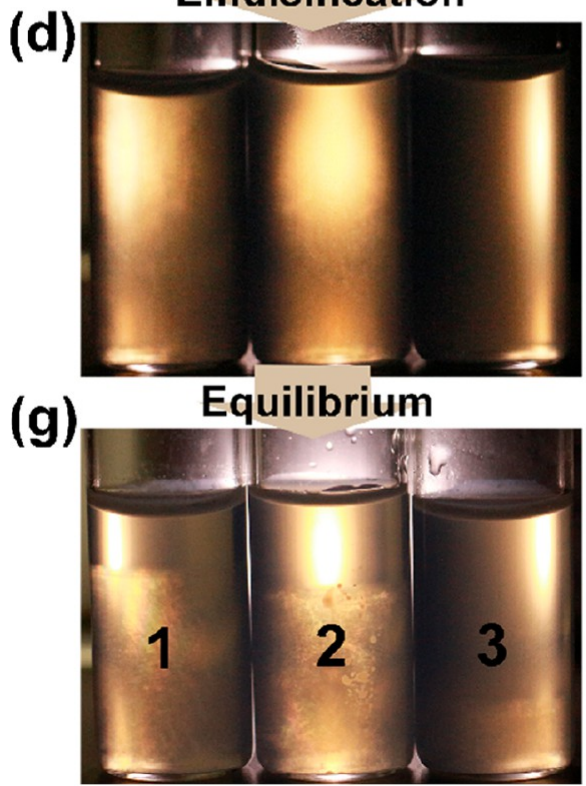

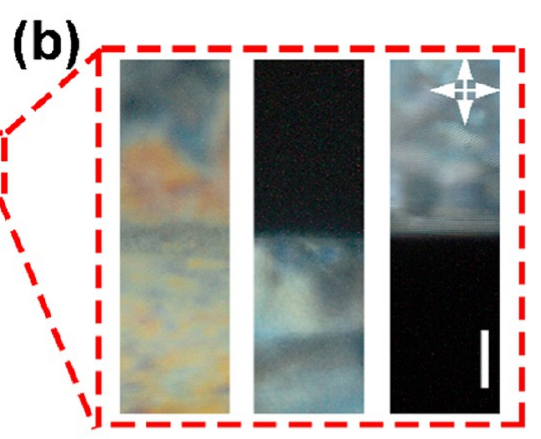

(e)

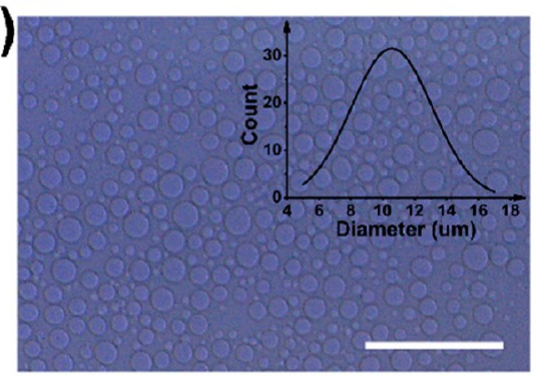

(h)

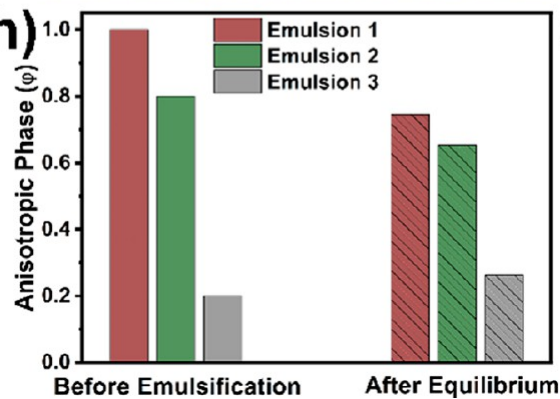

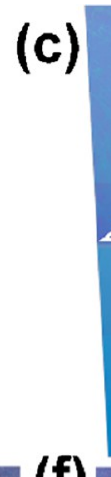

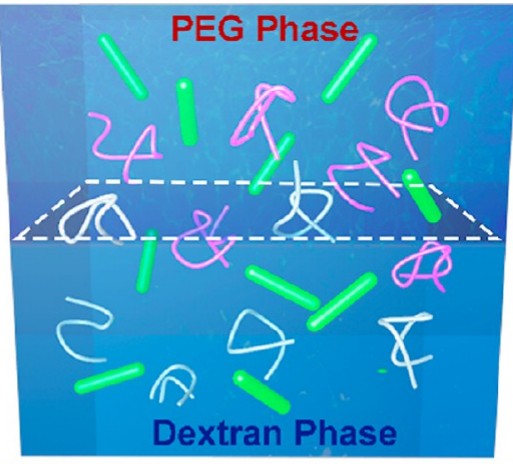

(f)
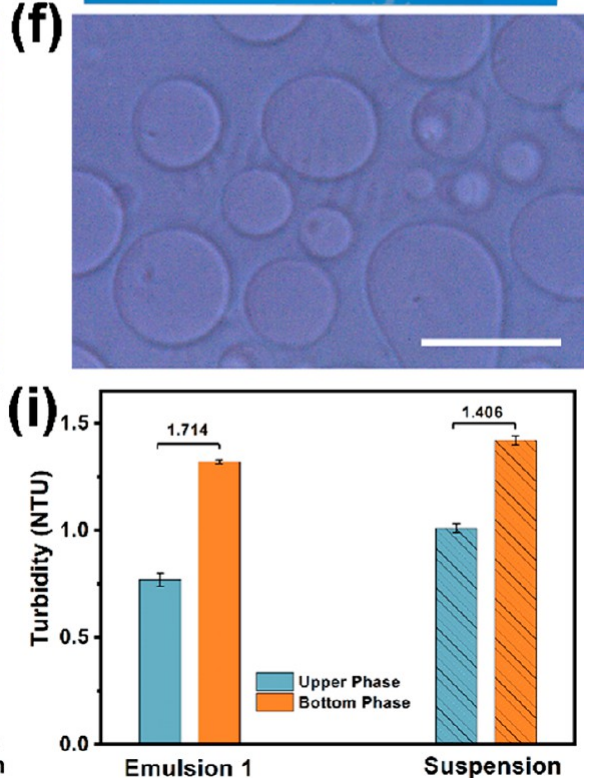

Figure 2. From macro to micro: binary water-in-water LC emulsion with anisotropy. (a) Photograph of demixed CNC/PEG (upper) and $\mathrm{CNC} /$ dextran (bottom) binary suspensions with clear phase boundary and different LC combinations. From left to right, cholesteric-tocholesteric $=1$, isotropic-to-cholesteric $=2$, and cholesteric-to-isotropic $=3$. The CNC concentration in the suspensions was 6 wt $\%$. $(\mathrm{b})$ POM images of the demixed suspensions that focused on the phase boundary, showing birefringent texture in cholesteric LC phase and optical extinction in the isotropic phase. (c) Schematic illustration of the water/water interface with gradient of polymer concentration. (d) Photograph of the dispersion obtained by vortex agitation, generating a homogeneous $\mathrm{W} / \mathrm{W}$ emulsion. Optical images of the prepared emulsions at (e) low and (f) high magnifications, showing polydisperse droplets with slightly nonspherical shape. The inset corresponds to the emulsion droplet size distribution. (g) Photograph of the prepared emulsions after equilibrium for 2 days, showing an upper isotropic phase and bottom anisotropic phase. (h) Calculated ratio of the anisotropic phase for emulsions 1-3, before and after emulsification. (i) Turbidity values of the separated emulsion 1 (cholesteric-to-cholesteric) and CNC suspension, upper and bottom phases, showing that the droplets were enriched in the bottom phase. The values in (i) were calculated from the turbidity ratio of the bottom and the upper phases. Scale bars: (b) $100 \mu \mathrm{m}$, (e) $100 \mu \mathrm{m}$, and (f) $20 \mu \mathrm{m}$.

potential of $-58.5 \mathrm{mV})$. We investigated the $\mathrm{CNC}$ phase separation in the presence of hydrophilic, coiled dextran and PEG (Figure 1a,b). As expected, no birefringence was seen for the pure CNC suspension at low particle concentration (3.0 wt $\%$ ), an isotropic system (Figure S2). By increasing the CNC concentration (e.g., $5.0 \mathrm{wt} \%)$, phase separation occurred into an upper isotropic and a bottom cholesteric phase. In the presence of either dextran or PEG, the CNC suspension separated into a biphasic system (Figure 1a). The volume fraction $(\varphi)$ of the cholesteric phase with respect to the initial suspension decreased from 0.689 (neat $\mathrm{CNC}$ suspension) to 0.237 (CNC/dextran) and $0.354(\mathrm{CNC} / \mathrm{PEG})$, corresponding to an increased $\mathrm{CNC}$ relative particle enrichment in the anisotropic phase (from 0.645 to 0.766 and 0.712 for the respective system as evaluated qualitatively by turbidimetry) (Figure $1 \mathrm{~b}$ and methods in Supporting Information). The broadening of the biphasic region and enrichment of CNC in the cholesteric phase resulted from the mutual exclusion effect between rodlike nanoparticles and the nonabsorbing polymer coils. ${ }^{33}$ Based on Flory's calculation, the anisotropic LC phase approaches the selectivity of a pure crystal in its rejection of a foreign component, whereas the isotropic counterpart can incorporate this component. ${ }^{49}$ Therefore, the addition of dextran or PEG into the biphasic suspension can replace the $\mathrm{CNC}$ in the isotropic phase and increase the rod particle concentration in the anisotropic phase, giving rise to an inhomogeneous suspension. The latter shows an uneven partition between the upper polymer-rich, CNC-poor phase and the bottom polymer-poor, CNC-rich phase. Compared to the biphasic system from neat $\mathrm{CNC}$ suspension, dispersing dextran or PEG into the fully anisotropic phase showed no apparent effect on phase separation (Figure S3), implying the polymer tolerance to the cholesteric LC.

Figure 1c-e shows the polarized optical microscopy (POM) images of the cholesteric LC before and after the addition of either dextran or PEG. The pure CNC cholesteric phase 
exhibited a long-range, continuous fingerprint texture with very few topological defects, characteristic of the helical arrangement over macroscopic distances. However, with the addition of either dextran or PEG, the helical pitch of the cholesteric phase was not only reduced (from 14.2 to 10.0 or $8.4 \mu \mathrm{m}$; see histograms in Figure S4) but also led to a fingerprint texture with a tortuous path and abundant disclinations and dislocations. The latter is related to the presence of the foreign polymer in the cholesteric phase, which is expected to produce internal constraints that could not be satisfied by the continuous strain of the LC director through bending, twisting, or splaying, creating more defects in the LC phase with low free energy at the defect cores. ${ }^{50}$ The average separation distance between adjacent $\mathrm{CNC}$ nanorods has been reported to range from 25 to $51 \mathrm{~nm}$ in the cholesteric phase, depending on the concentration; ${ }^{51}$ meanwhile, the measured hydrodynamic radii of coiled dextran and PEG are 10.2 and $8.3 \mathrm{~nm}$, respectively, smaller than the separation distance of CNC (Figure S5). Inasmuch as the size, shape, and relatively high concentration of polymer coils in the cholesteric phase, the polymers were inserted into the cholesteric structure and entered between CNCs (Figure S6), whereas any excess amount of polymers could induce distortions in cholesteric LC, with polymer enrichment inside the topological defects.

Water-in-Water Liquid Crystal Emulsion. When a $\mathrm{CNC} / \mathrm{PEG}$ suspension with either isotropic or cholesteric organizations was added into the $\mathrm{CNC} /$ dextran counterpart (at a volume ratio of 1:4), an aqueous system was created with a clear phase boundary at the water/water interface (Figure $2 a)$. Both phases contained the same concentration of polymer (10 wt \%) and with identical CNC concentration (6 wt \%), forming cholesteric-cholesteric, isotropic-cholesteric, or cholesteric-isotropic binary combinations. Phase demixing is explained by the repulsive interactions between PEG and dextran molecules, which have a small entropy of mixing, which is compensated by the positive mixing enthalpy in water, favoring segregation above critical concentrations. ${ }^{52}$ Magnified POM images of the PEG/dextran interfacial region exhibited a clear boundary with bright birefringent texture in the cholesteric phase and optical extinction in the isotropic counterpart (Figure 2b), confirming the demixing of the $\mathrm{CNC} /$ polymer suspensions. On the other hand, adding an isotropic $\mathrm{CNC} / \mathrm{PEG}$ suspension on top of another isotropic $\mathrm{CNC} /$ dextran suspension (CNC: $3 \mathrm{wt} \%$, polymer: $10 \mathrm{wt} \%$ ) led to a demixed profile between two isotropic counterparts. The bottom CNC/dextran phase became anisotropic after equilibrium, implying the diffusion of CNC from the PEG phase into the dextran phase (Figure S7). Although visual observation of the water/water interface in the $\mathrm{CNC} /$ polymer suspensions appears to be well-defined (macroscopic/microscopic scales), the water/water interface is in fact an interphase at the molecular level, for example, a layer that is much thicker compared to that of typical oil/water interfaces. ${ }^{53}$ Moreover, a polymer concentration gradient is expected to exist at the nanoscopic scale. ${ }^{54}$ At the interfacial zone, the total polymer concentration featured a local minimum owing to the repulsion between each polymer, which resisted interpenetration. The corresponding polymer concentrations varied gradually from the bulk value in the PEG phase to the bulk value in the dextran phase, across tens of nanometers (Figure 2c), ${ }^{11,55}$ the same order of distance between each individual CNC, allowing the $\mathrm{CNCs}$ at the interfacial zone to experience short-range repulsive interactions (across the water/water interface).
$\mathrm{W} / \mathrm{W}$ emulsions were prepared by emulsifying the respective binary aqueous $\mathrm{CNC}$ /polymer suspensions through vortexing (10 wt \% dextran and 10 wt \% PEG), showing a homogeneous appearance (Figure 2d). We first confirmed that the CNC/ PEG phase acted as the dispersed phase in the continuous $\mathrm{CNC} /$ dextran phase at the tested polymer concentrations, forming spherical CNC/PEG droplets (Figure S8). We then inspected the droplet size distribution and stability of the emulsion. A freshly prepared $\mathrm{W} / \mathrm{W}$ emulsion was sealed in a glass cuvette $(4 \mathrm{~cm} \times 1 \mathrm{~cm} \times 1 \mathrm{~mm})$ and kept undisturbed under ambient condition. Initially, the obtained emulsion exhibited polydispersed CNC/PEG droplets, with a relatively broad size distribution (Figure 2e). Magnified optical images showed spheroidal droplets, with average diameters ranging from 6 to $16 \mu \mathrm{m}$. Moreover, some fingerprint domains were observed inside and around the emulsion droplets, implying the self-assembling of CNC into a cholesteric organization (Figure 2f). The coalescence of dispersed droplets was relatively slow, with a gradual increase in droplet size during the first $5 \mathrm{~h}$. Small droplets $(<5 \mu \mathrm{m})$ merged together into larger ones (Figure S9 and Video S1). The stability behavior of the obtained W/W emulsion is in contrast to that of the typical Pickering stabilizing effect; namely, the cellulose nanoparticles in the $\mathrm{W} / \mathrm{W}$ emulsions freely assembled across the PEG/ dextran interfacial zone.

After standing for 2 days, the as-prepared $\mathrm{W} / \mathrm{W}$ emulsions eventually demixed into two phases, with an upper clear isotropic phase and a bottom turbid anisotropic phase (Figure $2 \mathrm{~g}$ and Figure S10). It should be noted that the droplet size and distribution of the demixed emulsions after 7 days (Figure S11) showed little change compared with the freshly prepared samples (Figure 2e,f), indicating a stable $\mathrm{W} / \mathrm{W}$ system. We note that 2 days were already sufficient to ensure a fully demixed emulsion into a biphasic system (see the inset in Figure S11b). Herein, the emulsions mixed using different LC phase combinations, cholesteric (PEG)-to-cholesteric (dextran), isotropic (PEG)-to-cholesteric (dextran), and cholesteric (PEG)-to-isotropic (dextran), are referred to as emulsion 1, 2, and 3 , respectively. It is worth mentioning that all of the emulsions separated into two coexisting phases, similar to the initial biphasic CNC suspension with individual guest polymers. To quantify the emulsion phase separation, the volume fraction of anisotropic phase, $\varphi$, was calculated before emulsification and after complete phase separation. The values of $\varphi$ decreased from 1 to 0.746 and 0.8 to 0.653 for emulsions 1 and 2 but increased from 0.2 to 0.263 for emulsion 3 . These results suggest that, in the presence of the two hydrophilic polymers, a newly formed equilibrated system ("re-equilibrium") occurred in the CNC cholesteric assembly (Figure $2 \mathrm{~h})$. The droplet distributions in the two phases were followed by measuring the turbidity and by using regular optical image counting. All of the emulsions and initial CNC suspensions showed larger turbidity in the bottom phase, whereas the upper phase was translucent (Figure S12). The relative turbidity ratio of the bottom phase to the upper phase for emulsion 1 and initial CNC suspension was 1.714 and 1.406, respectively, indicating a significant enrichment of the droplets in the emulsion bottom phase as the former was more turbid than the binary CNC suspension (Figure 2i). Furthermore, the measured turbidity ratio for emulsions 2 and 3 was 1.568 and 1.792, respectively, and both followed a similar trend as emulsion 1 (Figure S12). The difference of emulsion droplet distribution in the upper isotropic and bottom cholesteric 

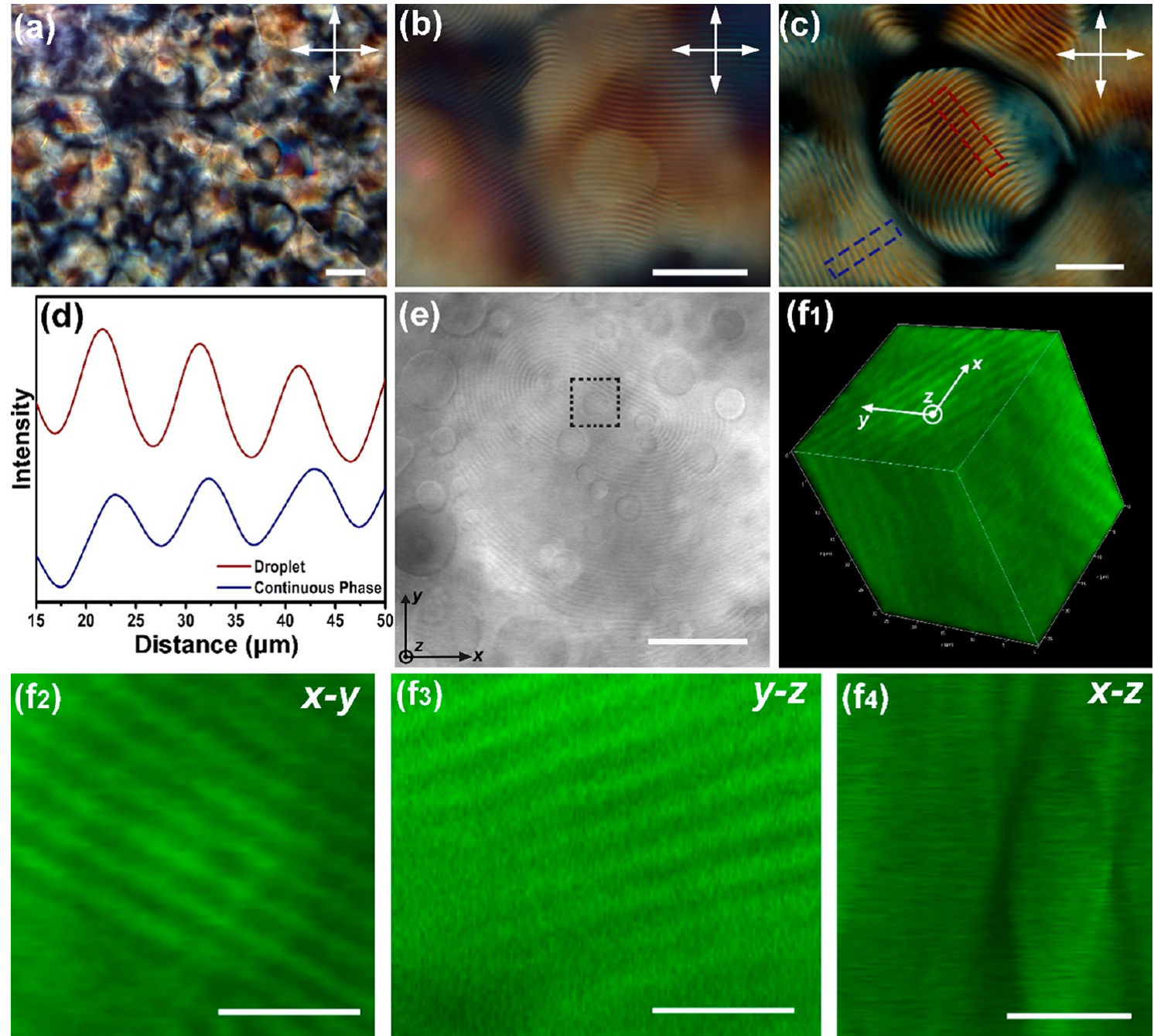

(g) Small PEG droplet
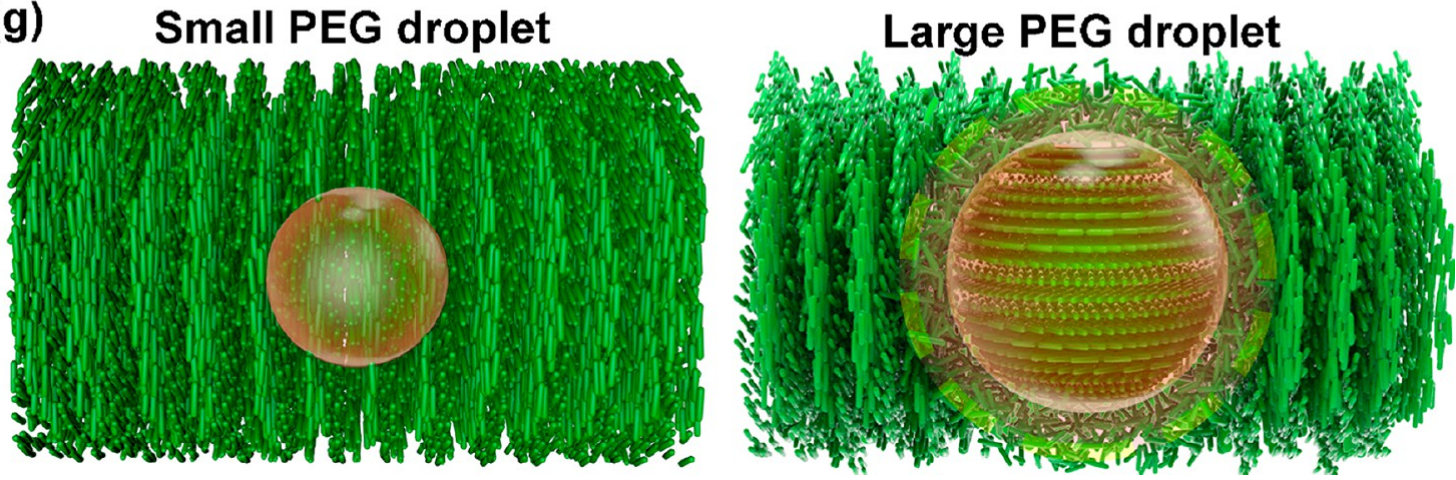

Figure 3. Hierarchical LC-in-LC emulsion with global cholesteric organization. (a) POM image of the cholesteric W/W emulsion at low magnification. High-magnification POM images of the W/W emulsions with (b) small and (c) large CNC/PEG droplet in CNC/dextran continuous phase. (d) Image analysis of the cholesteric W/W emulsion, showing the same fluctuations of helical pitch in the dispersed phase (droplet) and the continuous phase. (e) Polarized CLSM of cholesteric W/W emulsion droplets. The black dashed square highlights the droplet used for 3D reconstruction. (f) Reconstructed 3D structure and image planes $(x-y, y-z$, and $x-z)$ from polarized CLSM of the suspended droplet in the $\mathrm{W} / \mathrm{W}$ emulsion (e), demonstrating its internal cholesteric structure. $(\mathrm{g})$ Schematic illustration showing the $\mathrm{W} / \mathrm{W}$ LC emulsion with global organization, showing interconnected CNC assemblies with uniform cholesteric ordering across a small PEG droplet (upper) and distortions around a large PEG droplet (bottom). Scale bar: (a-c,e) $50 \mu \mathrm{m}$ and (f) $10 \mu \mathrm{m}$.

phases was further confirmed by optical imaging (Figure S13), which indicated that the suspended droplets were preferably present in the denser LC phase rather than the upper isotropic phase.

Previous reports have suggested that the uneven partitioning of rigid foreign colloids into LCs incurs an elastic energy penalty and LC director deformations in the bulk phase; ${ }^{37,56,57}$ that is, partitioning droplets into the isotropic phase is more energy preferable compared to the cholesteric phase. However, our experiments indicate the opposite effect. One possible reason that explains the difference may be related to the large droplet size and density difference between the droplets and 

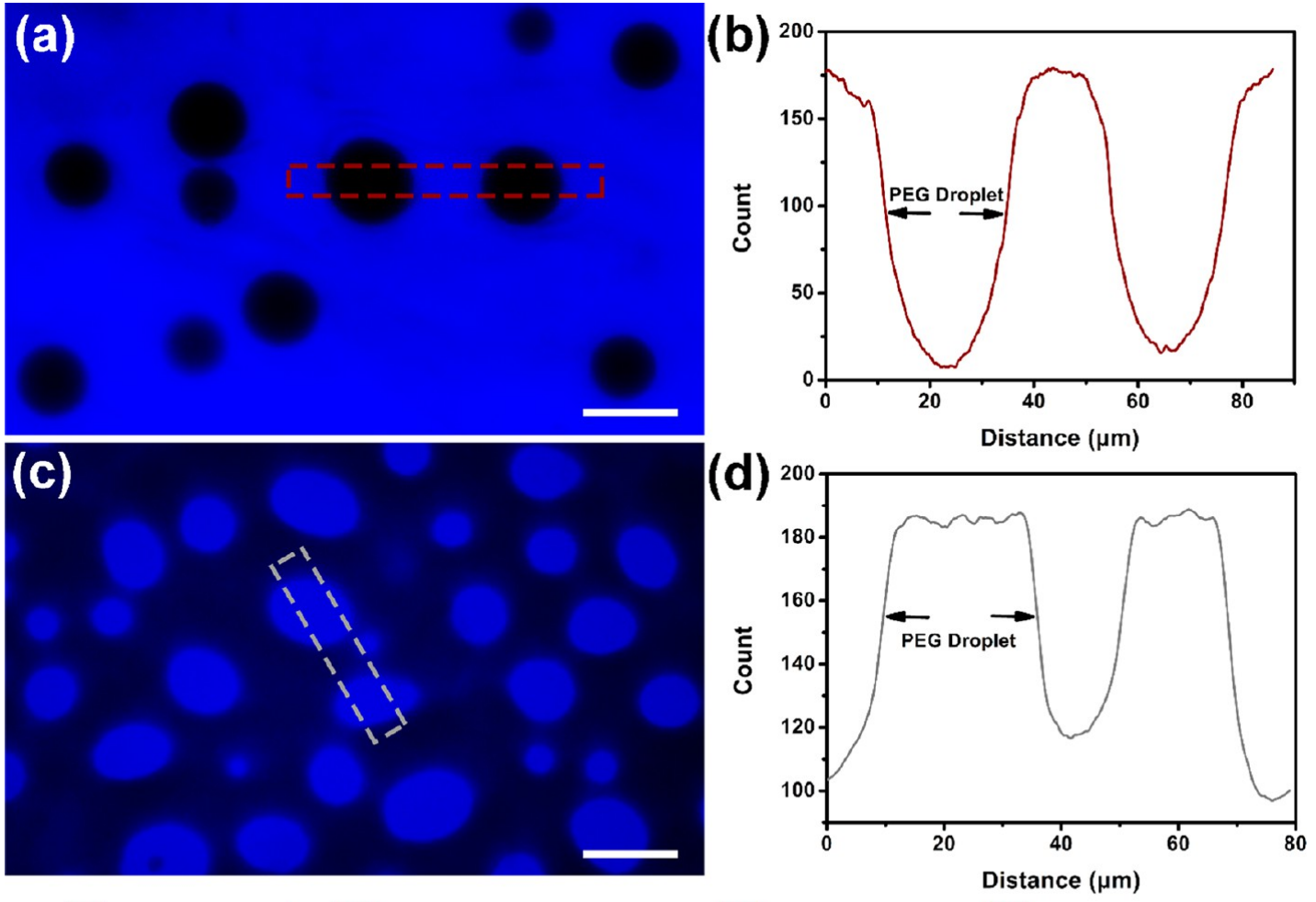

ПCNC-PEG $>$ ПCNC-Dextran
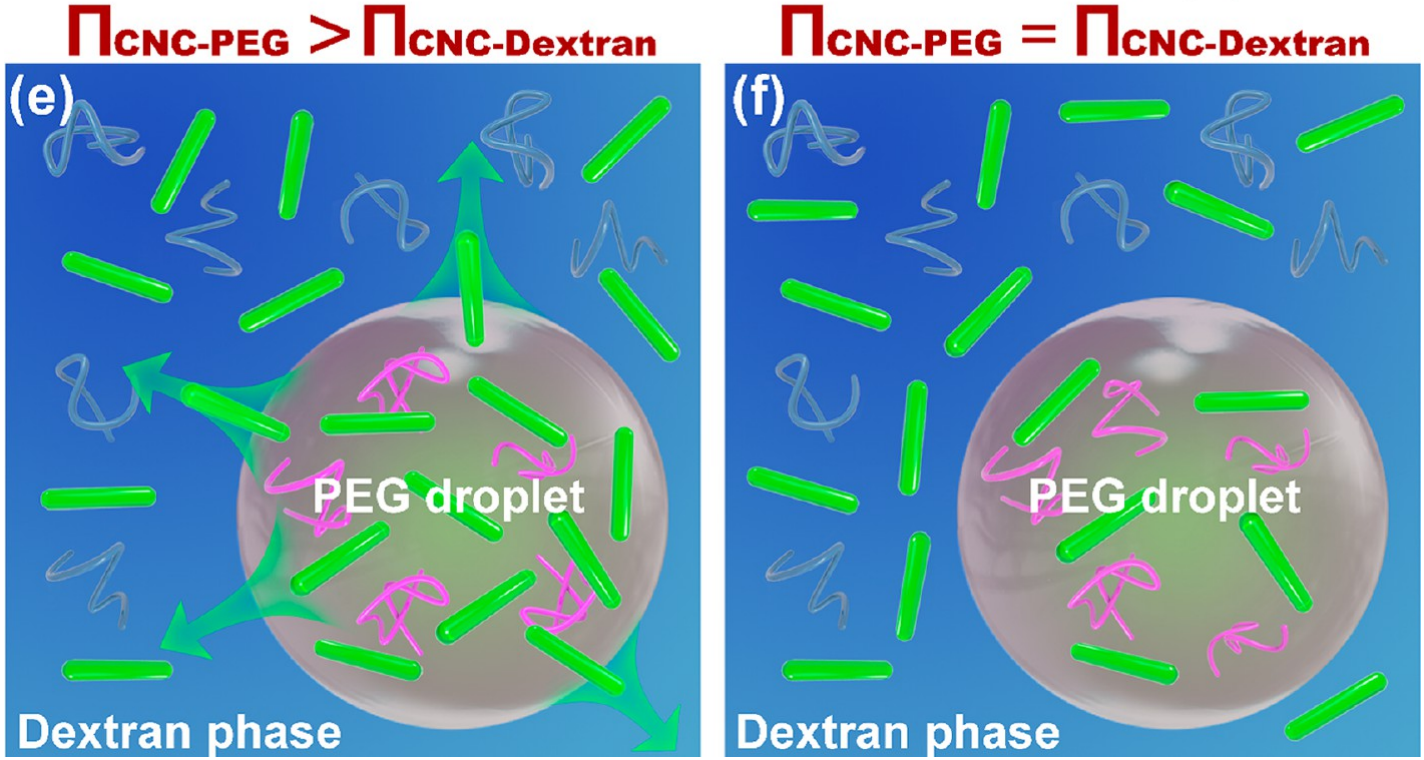

Figure 4. Osmotic-driven target transfer of $\mathrm{CNC}$ across the water/water interface. (a) Fluorescence microscopy image and (b) relative intensity analysis of a W/W emulsion with the same concentration of PEG and dextran (both at 10 wt \%) and with fluorescently labeled CNC initially added in the PEG phase. (c) Fluorescence image and (d) relative intensity analysis of a W/W emulsion with a lower PEG concentration in the dispersed phase (PEG: 5 wt \%, dextran: $10 \mathrm{wt} \%)$. (e,f) Schematic presentation of the osmotic pressure-induced target transfer of CNC between PEG droplet and continuous dextran phase. The case illustrated in (e) is more dominant in the emulsions with $10 \%$ PEG, whereas that in (f) indicates a lower diffusion gradient, as was the case of the emulsion produced with PEG at lower concentration. Scale bar: $20 \mu \mathrm{m}$.

the surrounding continuous phase, given that gravity acts on the droplets and dominates over buoyancy. Another reason can be related to the possibility of a permeable and elastic PEG/ dextran droplet interface, which allows $\mathrm{CNC}$ diffusion while being capable of deforming at the isotropic/cholesteric interface. As such, Figure $3 a-c$ shows POM images of the bottom phase of emulsion 1 with $\mathrm{W} / \mathrm{W}$ organization. At low magnification, the emulsion exhibited bright birefringent textures and curved dark brushes that corresponded to the typical topological defects and extinction orientation of the cholesteric LC. ${ }^{50}$ The presence of defects as well as the extinction of $\mathrm{CNC}$ director in the LC emulsion was further confirmed by removing the cross-polarizers (Figure S14), indicating the existence of polymer molecules and volume distortion of a droplet that disturb the orientation of the cholesteric LC phase. In addition, suspended droplets were densely packed with distorted spherical shapes, indicating the enrichment of droplets in the bottom phase and elastic water/ 
water interfaces. High-magnification POM images demonstrate a long-range fingerprint texture around and inside the water droplets, characteristic of the CNC cholesteric arrangement. ${ }^{31}$ It should be noted that small water droplets showed uniform fingerprint textures across the boundary between PEG droplets and the dextran continuous phase, which implied short-range interconnected CNC assemblies (Figure 3b). In contrast, the fingerprint textures in large droplets were confined and separated by PEG droplets with a circle of line defects around, indicating different CNC orientations in the surrounding continuous dextran phase and the PEG droplets (Figure 3c). The helical pitch of CNC cholesteric LC in both configurations showed no distinct differences in the frequency of optical intensity between PEG and dextran phases (Figure $3 d$ ), indicating the LC-in-LC global cholesteric organization of CNC through a barricade comprising the PEG/dextran interface. To further demonstrate the droplet structure, we performed nonintrusive polarized confocal laser scanning microscopy (CLSM) at different depths across the droplet ( $z$-stack) and to visualize the 3D structure and LC ordering of the droplets in the $\mathrm{W} / \mathrm{W}$ emulsions (Figure $3 \mathrm{e}, \mathrm{f}$ and Video S2). The reconstructed 3D structure of the droplet and corresponding CLSM images at different image planes provide clear evidence confirming the internal structure of the obtained LC-in-LC emulsion (i.e., a cholesteric-in-cholesteric system).

Based on the information above, Figure $3 \mathrm{~g}$ provides a schematic illustration (not to scale) of the CNC cholesteric assembly in W/W LC emulsions. The assembly behavior of LC-in-LC emulsions greatly depends on the size of the droplet. ${ }^{58}$ At given high volume fraction, the emulsion droplets become densely packed and deform from spheres into curved polyhedra. When the suspended water droplets are small, entropy factors play a dominant role in the assembly of LC-inLC emulsion; namely, the droplets are confined into the subregions of the cholesteric phase, without large distortion of the surrounding LC director. In contrast, when a large PEG droplet is embedded inside the cholesteric matrix, the suspended droplet breaks the spatial ordering of the surrounding LC director, resulting in a volume-induced distortion around the droplet.

Permeable Assembly of Liquid Crystal Colloids. As noted above, both PEG and dextran allow CNC assemblies in the respective aqueous suspensions, acting as a ternary system comprising water, rodlike CNCs, and the respective coiled polymer. Mixing the two immiscible polymer suspensions yields $\mathrm{W} / \mathrm{W}$ emulsions, phase separation, and eventually reequilibrium of the CNC cholesteric assembly. From the above observation, a question that naturally arises is how the CNCs reassemble during the emulsifying process. By using Calcofluor white stain to bind to $1-4 \beta$-glycosidic bonds on the CNC surface, ${ }^{59}$ we selectively labeled the CNC in the PEG phase as the fluorescent marker; meanwhile, the $\mathrm{CNC}$ in the continuous dextran phase was not tagged (see Supporting Information). Figure $4 \mathrm{a}, \mathrm{b}$ shows the fluorescent image and corresponding intensity analysis of a freshly prepared $\mathrm{W} / \mathrm{W}$ emulsion with dye-labeled CNC only in the PEG phase (10 wt \% dextran and 10 wt \% PEG). Surprisingly, when the two $\mathrm{CNC} /$ polymer suspensions were brought into contact, the $\mathrm{CNC} /$ dextran continuous phase appeared fluorescent, whereas the PEG droplets were less fluorescent. Evaluation of the fluorescent intensity maxima along a line across the emulsion droplet showed high fluorescence intensity in the continuous dextran phase, and low fluorescence was registered in the droplet area, which implies an uneven partitioning of the labeled CNC in the $\mathrm{W} / \mathrm{W}$ emulsion and target transfer of CNC from the PEG phase to the dextran phase (Figure $4 b$ ). We note that when the PEG concentration in the dispersed phase was reduced to $5 \mathrm{wt}$ $\%$, while keeping the dextran concentration at $10 \mathrm{wt} \%$, a more balanced fluorescence intensity between the phases was observed. This latter result indicates that compared to the previous case of $10 \mathrm{wt} \%$ PEG, the PEG phase at lower concentration held a higher number density of dyed CNC (Figure 4c). Moreover, further reduction of PEG concentration to $2 \mathrm{wt} \%$ generated a soluble PEG/dextran suspension (Figure S15). As the emulsions at different PEG concentrations ( 5 or 10 wt \%) have been identified as PEG-in-dextran type (Figure $\mathrm{S} 8$ ), the above results suggest a concentrationinduced transfer of CNC across the water/water interface: at high PEG concentration, CNC in the dispersed PEG phase diffuses into the continuous dextran phase, whereas diffusion is suppressed at low concentration.

Figure 4e,f illustrates our suggested diffusion of CNCs upon mixing. As PEG and dextran are uncharged polymers, compared to the bulk phase, the interphase around the droplets exhibits a gradient of polymer concentration, as far as the relative composition, leading to an interfacial polymer/ polymer barrier that results from entropy-induced repulsive interactions between the polymer molecules. ${ }^{6}$ In this case, the interfacial region in the $\mathrm{W} / \mathrm{W}$ emulsion is somewhat analogous to a permeable polymer membrane. Osmosis occurs in the presence of the two aqueous phases of different solute concentrations, ${ }^{60}$ in which the corresponding osmotic pressure in each phase can be calculated as $\Pi=c_{\mathrm{P}} R T+c_{\mathrm{CNC}} R T$, where $\Pi$ is the osmotic pressure, $C_{\mathrm{P}}$ and $C_{\mathrm{CNC}}$ are the molar concentration of polymer (PEG and dextran, respectively) and $\mathrm{CNC}, R$ is the ideal gas constant, and $T$ is the temperature. As the initial concentrations of $\mathrm{CNC}$ in both aqueous phases are the same, the osmotic pressure mainly depends on the molar concentration of PEG and dextran. Due to the differences in molecular weight of PEG and dextran $\left(2 \times 10^{5}\right.$ and $4.5 \times 10^{5}$, respectively), at the same polymer mass fraction (10 wt \%), $c_{\mathrm{PEG}}$ is larger than $c_{\text {dextran; }}$ as a consequence, $\Pi_{\mathrm{CNC} / \mathrm{PEG}}>$ $\Pi_{\mathrm{CNC} / \text { dextran. }}$. When the two $\mathrm{CNC} /$ polymer aqueous suspensions are brought directly into contact, $\mathrm{CNC}$ nanoparticles are transferred through the water/water interface, from the PEG phase of relatively higher osmotic pressure into the dextran phase of lower osmotic pressure. This occurs given the fact that polymer molecules in each phase are not subjected to crossdiffusion. The transfer of CNC continues until the osmotic pressures in the two phases are balanced, resulting in CNC redistribution and re-equilibration into cholesteric organization through the water/water interface. When the polymer concentration in the dispersed PEG phase is reduced, a lower gradient between the two phases inhibits the osmoticinduced CNC transfer, producing an effective lockdown of $\mathrm{CNC}$ in their original polymer (PEG) phase. Moreover, because of the different polymer osmotic contributions in each phase, the diffusing cellulose nanoparticles are unevenly partitioned in the suspended droplets and surrounding continuous phase, generating distortions and defects in the cholesteric LC phase.

In order to further confirm our hypothesis of the permeable interface in $\mathrm{W} / \mathrm{W}$ emulsions, we dispersed cholesteric $\mathrm{CNC} /$ PEG phase into isotropic CNC/dextran phase (both PEG and dextran were 10 wt \%) and monitored their self-assembly process through POM imaging. Initially, the polydispersed 


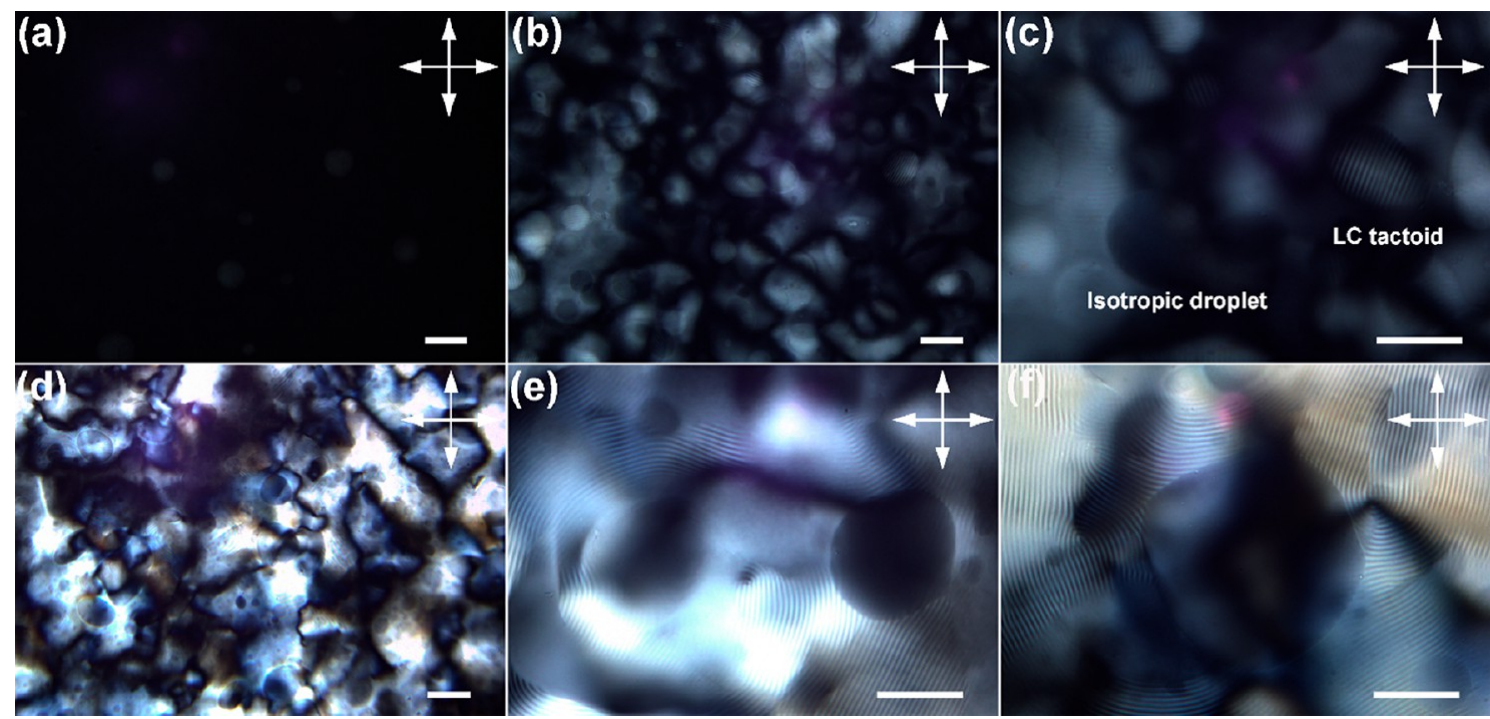

Figure 5. Optical tracking of the CNC reassembly in W/W emulsion. (a) Low-magnification POM image of the freshly prepared W/W emulsion with isotropic continuous CNC/dextran phase and cholesteric CNC/PEG droplets. (b) and (c) POM images of the equilibrated $\mathrm{W} / \mathrm{W}$ emulsion with isotropic CNC/PEG droplet and cholesteric tactoid in the continuous CNC/dextran phase. (d-f) POM images of the $\mathrm{W} / \mathrm{W}$ emulsion with isotropic CNC/PEG droplet and cholesteric organized CNC/dextran continuous phase. Scale bar: $50 \mu \mathrm{m}$.

$\mathrm{CNC} / \mathrm{PEG}$ droplet showed an anisotropic texture, whereas the surrounding $\mathrm{CNC} /$ dextran phase appeared isotropic, which was attributed to the demixing of the polymer solutions (Figure 5a). In the few hours that followed, we distinguished some spherical and spindle-shaped birefringent tactoids with fingerprint textures nucleated in the isotropic $\mathrm{CNC} /$ dextran phase, whereas the previous cholesteric CNC/PEG droplet turned isotropic instead (Figure 5b,c). The formation of tactoids in an isotropic phase requires the increase of the particle concentration and gathers together additional particles into a microdroplet domain with LC ordering. Target transfer of $\mathrm{CNC}$ from the cholesteric $\mathrm{CNC} / \mathrm{PEG}$ phase into the isotropic $\mathrm{CNC} /$ dextran phase via diffusion could break the balance in the isotropic phase and give rise to the reassembly of $\mathrm{CNC}$ in $\mathrm{W} / \mathrm{W}$ emulsions. On the contrary, dispersing an isotropic $\mathrm{CNC} / \mathrm{PEG}$ suspension into the cholesteric CNC/ dextran continuous phase generated a W/W emulsion with isotropic-in-cholesteric organization, and the large area of topological defects in the continuous cholesteric phase was ascribed to the diffusion of CNC (Figure $5 \mathrm{~d}-\mathrm{f}$ ). All in all, the POM results suggest the osmotic pressure-driven permeable self-assembly of CNC between the dispersed PEG phase and continuous dextran phase.

\section{CONCLUSIONS}

In summary, we employed an immiscible polymer dispersed system to produce $\mathrm{W} / \mathrm{W}$ emulsions with permeable CNC selfassembly and global LC organization. The demixed PEG and dextran solutions host the cholesteric LC in both the dispersed and continuous phases, providing useful physical insights to guide the coassembly of the $\mathrm{CNC}$ and polymer. Target transfer of CNC occurs across the water/water interface according to the balance of osmotic pressure gradients between the two polymer phases, resulting in a LC emulsion with hierarchical cholesteric-in-cholesteric ordering. These $\mathrm{W} / \mathrm{W}$ emulsions can serve as a versatile platform for fundamental studies, seeking to understand the interplay and target transport of colloidal particles across immiscible phases. We believe that this simple but robust emulsion system can be extended to other colloidal particles with varying anisotropies (1D nanorods, 2D nanosheets, and 3D polyhedral particles, etc.), creating a series of $\mathrm{W} / \mathrm{W}$ LC emulsions that can be useful in a variety of applications.

\section{EXPERIMENTAL SECTION}

Materials and Instruments. Cellulose nanocrystal gel (10 wt \%) was obtained from the U.S. Forest Products Laboratory at the University of Maine. The CNC was prepared by sulfuric acid hydrolysis of dissolving-grade wood fibers. After dilution and membrane filtration, the resulting system consisted of a concentrated suspension of CNC that had typical dimensions of $\sim 10-15 \mathrm{~nm}$ in width and $\sim 300-400 \mathrm{~nm}$ in length and contained 0.95 wt \% sulfur. Dextran $\left(M_{\mathrm{w}}=4.5 \times 10^{5}\right)$, polyethylene glycol $\left(\mathrm{PEG}, M_{\mathrm{w}}=2 \times 10^{5}\right)$, and Calcofluor white stain were purchased from Sigma-Aldrich (Helsinki, Finland). All chemicals were used as received without further purification. Milli-Q water (18 M $\Omega$ ) was obtained from a Millipore water system and used throughout the experiments.

Polarized optical microscope imaging was conducted with an Olympus BX53-P microscope (Olympus, Japan), with images taken by polarizers in a perpendicular arrangement to verify the anisotropy of the emulsion samples. For visualizing the boundary bewteen two phases with POM, an optical quartz absorption cuvette $(1 \mathrm{~mm}$ path length, Hellma, Germany) was used. Fluorescent microscope imaging was conducted with a Zeiss Axio Observer optical microscope (Zeiss, Germany) equipped with $40 \times$ or $63 \times$ objectives to visualize dyelabeled CNC in the emulsion samples. A drop of freshly dyed emulsion sample was placed on a microscope slide and covered with a glass coverslip (Assistent, Sondheim, Germany), which was quickly fixed by nail polish to avoid evaporation. The excitation/emission spectra for dyed CNCs were 365 and $435 \mathrm{~nm}$, respectively. Optical microscope imaging was performed with a Leica DM 750 (Leica, Germany) with $40 \times$ and $20 \times$ objective lens to track the distribution and in situ merging of the emulsion droplets. The measured POM, fluorescent and optical images were further analyzed through a noncommercial ImageJ software. ${ }^{61}$ Polarized confocal laser scanning microscopy was performed with a Leica SP5 microscope (Leica) in the absence of fluorescent labeling. A $488 \mathrm{~nm}$ argon laser was used as the light source combined with a $40 \times$ oil immersion objective to image the sample. The optical birefringence was collected in the transmission mode using a polarized laser and a linear polarized filter oriented perpendicular to the polarization plane of the incident light. 
The focused laser scanned the sample at a fixed depth and created a horizontal optical slice. By repeating the scans at different depths, we obtained a stacking of each optical slice ( $z$-stack), generating a polarized 3D pattern. ${ }^{23}$ The images were analyzed using Leica Application Suite X (LAS X). The optical transmittance of suspensions and emulsions was measured using a UV-2550 UV-vis spectrophotometer (SHIMADZU, Kyoto, Japan) operated at room temperature. The sample was loaded in an optical quartz cuvette (1 $\mathrm{cm}$ path length, Hellma, Germany). All measurements were carried out by duplicate with freshly prepared samples.

Preparation of CNC/PEG and CNC/Dextran Suspensions. Initially, concentrated CNC gel was diluted to $200 \mathrm{~mL}$ by adding Milli-Q water, generating a homogeneous suspension with a concentration of 5.0 or $6.0 \mathrm{wt} \%$. Then, the resulting suspension was sonicated for $2 \mathrm{~min}$ in an ice bath and sealed in a $300 \mathrm{~mL}$ bottle. After standing for 2 days, the resulting suspension was separated into two phases, an upper isotropic phase and a bottom cholesteric phase.

$\mathrm{CNC} / \mathrm{PEG}$ and $\mathrm{CNC} /$ dextran suspensions were prepared by dissolving polymers into diluted CNC suspension (5 wt \%) after sonication. After given amounts of polymer were added into the CNC suspension, the obtained mixture was vigorously stirred overnight at room temperature and left to stand for 2 days, eventually separating in a two-phase system.

Preparation of Water-in-Water Liquid Crystal Emulsion. To investigate the liquid crystal nature of $\mathrm{CNC}$ in the $\mathrm{W} / \mathrm{W}$ emulsions, the separated upper isotropic and bottom cholesteric phases of diluted CNC suspensions (6 wt \%) were used to dissolve dextran (10 wt \%) and PEG $(2,5$, or $10 \mathrm{wt} \%)$. After dissolution and storage, the isotropic and reseparated cholesteric phases, containing the polymers, were collected for further use.

The PEG-in-dextran W/W emulsions were prepared by mixing all components using a vortex (Scientific Industries, NY, USA) operated at a level of 8 for $30 \mathrm{~s}$. The PEG-to-dextran volume ratio was 20/80 for all of the samples. The order of mixing was found to have no effect on emulsion behavior. The emulsions prepared with CNC suspensions would undergo separation during storage into an upper isotropic phase and a bottom cholesteric phase. The emulsion behavior was monitored after storage at room temperature for 7 days. The emulsions of different types were prepared using the isotropic $\mathrm{CNC}$ /polymer and cholesteric $\mathrm{CNC} /$ polymer suspensions (isotropic $\mathrm{CNC} / \mathrm{PEG}$ in cholesteric $\mathrm{CNC} /$ dextran, cholesteric $\mathrm{CNC} / \mathrm{PEG}$ in isotropic $\mathrm{CNC} /$ dextran, cholesteric $\mathrm{CNC} / \mathrm{PEG}$ in cholesteric $\mathrm{CNC} /$ dextran, and isotropic $\mathrm{CNC} / \mathrm{PEG}$ in isotropic $\mathrm{CNC} /$ dextran emulsion)

To visualize the PEG phase in the emulsion, the CNC in PEG was dyed with Calcofluor white stain prior to emulsion preparation. Briefly, $10 \mu \mathrm{L}$ of Calcofluor white stain aqueous solution was added into a $5 \mathrm{~mL}$ CNC/PEG suspension (10 wt \%) under vigorous stirring to generate a homogeneous dye-labeled CNC/PEG suspension. The $\mathrm{W} / \mathrm{W}$ emulsion containing dyed CNCs was prepared following the above procedure, and the stained emulsions were stored at room temperature before observation via a fluorescence microscope.

Calculation of the Turbidity. The optical turbidity (at $600 \mathrm{~nm}$ ) of the separated upper and bottom phases of CNC suspensions and emulsions were determined using the following equation: ${ }^{62}$

$$
\text { turbidity }=2.3 \times \log \frac{1}{T}
$$

where $T$ is the transmittance. All calculations were averaged from data obtained by testing freshly prepared duplicate samples.

\section{ASSOCIATED CONTENT}

\section{(s) Supporting Information}

The Supporting Information is available free of charge at https://pubs.acs.org/doi/10.1021/acsnano.0c05251.

TEM image of CNCs; photograph and POM images of 3 wt \% CNC suspensions; photographs of CNC/PEG and $\mathrm{CNC} /$ dextran cholesteric liquid crystal phases; histogram of helical pitch of the suspensions; hydrodynamic radii of PEG and dextran solutions; schematic illustration of the inset of polymer coils between cholesteric liquid crystal planes; POM images of the phase transition in phase-separated isotropic CNC/PEG and isotropic $\mathrm{CNC} /$ dextran suspensions; confocal images of water-in-water (W/W) emulsion droplets; optical images of emulsion droplet merging and droplet uneven distribution; POM images of separated W/W emulsions; optical images of the droplet after 7 day storage; UV-vis spectra of emulsions and suspensions; optical images of $\mathrm{W} / \mathrm{W}$ emulsions with and without cross-polarizer; fluorescent image of emulsion with $2 \mathrm{wt}$ \% PEG and 10 wt \% dextran in CNC suspensions (PDF)

Video S1: Slow merging and growing of water-in-water emulsion droplets during equilibration (MP4)

Video S2: $z$-Stacking of the polarized CLSM images with different depths which focus on the droplet arrays (MP4)

\section{AUTHOR INFORMATION}

\section{Corresponding Authors}

Guang Chu - Bio-based Colloids and Materials, Department of Bioproducts and Biosystems, School of Chemical Engineering, Aalto University, FI-00076 Aalto, Espoo, Finland; ○ orcid.org/0000-0003-1538-5276; Email: chuguang88@ gmail.com

Orlando J. Rojas - Bioproducts Institute, Departments of Chemical \& Biological Engineering, Chemistry, and Wood Science, The University of British Columbia, Vancouver, BC V6T 1Z3, Canada; Bio-based Colloids and Materials, Department of Bioproducts and Biosystems, School of Chemical Engineering, Aalto University, FI-00076 Aalto, Espoo, Finland; ○ orcid.org/0000-0003-4036-4020; Phone: +1-604-822-

3457; Email: orlando.rojas@ubc.ca, orlando.rojas@aalto.fi

\section{Authors}

Long Bai - Key Laboratory of Bio-based Material Science and Technology of Ministry of Education, College of Material Science and Engineering, Northeast Forestry University, Harbin, Heilongjiang 150040, P.R. China; Bioproducts Institute, Departments of Chemical \& Biological Engineering, Chemistry, and Wood Science, The University of British Columbia, Vancouver, BC V6T 1Z3, Canada; 이이.org/0000-00033356-9095

Siqi Huan - Key Laboratory of Bio-based Material Science and Technology of Ministry of Education, College of Material Science and Engineering, Northeast Forestry University, Harbin, Heilongjiang 150040, P.R. China; Bioproducts Institute, Departments of Chemical \& Biological Engineering, Chemistry, and Wood Science, The University of British Columbia, Vancouver, BC V6T 1Z3, Canada; 이이이.org/0000-00031688-9484

Bin Zhao - Bio-based Colloids and Materials, Department of Bioproducts and Biosystems, School of Chemical Engineering, Aalto University, FI-00076 Aalto, Espoo, Finland

Ya Zhu - Bio-based Colloids and Materials, Department of Bioproducts and Biosystems, School of Chemical Engineering, Aalto University, FI-00076 Aalto, Espoo, Finland

Jordi Esquena - Institute of Advanced Chemistry of Catalonia, Spanish National Research Council (IQAC-CSIC) and Networking Research Center on Bioengineering, Biomaterials 
and Nanomedicine (CIBER-BBN), Barcelona 08034, Spain; (1) orcid.org/0000-0002-9188-5259

Feng Chen - Bio-based Colloids and Materials, Department of Bioproducts and Biosystems, School of Chemical Engineering, Aalto University, FI-00076 Aalto, Espoo, Finland; (1) orcid.org/0000-0002-9867-5633

Guang Gao - Department of Cellular and Physiological Sciences, Life Science Institute, The University of British Columbia, Vancouver, BC V6T 1Z3, Canada

Eyal Zussman - NanoEngineering Group, Faculty of Mechanical Engineering, Technion-Israel Institute of Technology, Haifa 3200003, Israel; 이이이.org/0000-0002-4310-6548

Complete contact information is available at:

https://pubs.acs.org/10.1021/acsnano.0c05251

\section{Author Contributions}

${ }^{\#}$ L.B. and S.H. contributed equally to this work.

\section{Notes}

The authors declare no competing financial interest.

\section{ACKNOWLEDGMENTS}

This work was a part of the Academy of Finland's Flagship Programme under Project Nos. 318890 and 318891 (Competence Center for Materials Bioeconomy, FinnCERES). The authors also acknowledge support by the Canada Excellence Research Chair initiative, the Canada Foundation for Innovation (CFI), and the European Research Council under the European Union's Horizon 2020 research and innovation program (ERC Advanced Grant Agreement No. 788489, “BioElCell”). J.E. acknowledges the i-LINK1188 collaborative project, the AIE founding agency and the "Grupo de Nanotecnologia Farmacéutica" UB-CSIC Associated Unit. Polarized CLSM imaging was performed in the LSI IMAGING facility of the Life Sciences Institute of the University of British Columbia using infrastructure funded by BC Knowledge Development Fund as well as a Strategic Investment Fund (Faculty of Medicine, University of British Columbia).

\section{REFERENCES}

(1) Bai, L.; Huan, S. Q.; Xiang, W. C.; Rojas, O. J. Pickering Emulsions by Combining Cellulose Nanofibrils and Nanocrystals: Phase Behavior and Depletion Stabilization. Green Chem. 2018, 20, $1571-1582$.

(2) McClements, D. J.; Bai, L.; Chung, C. Recent Advances in the Utilization of Natural Emulsifiers to Form and Stabilize Emulsions. Annu. Rev. Food Sci. Technol. 2017, 8, 205-236.

(3) Bai, L.; Greca, L. G.; Xiang, W.; Lehtonen, J.; Huan, S.; Nugroho, R. W. N.; Tardy, B. L.; Rojas, O. J. Adsorption and Assembly of Cellulosic and Lignin Colloids at Oil/Water Interfaces. Langmuir 2019, 35, 571-588.

(4) Esquena, J. Water-in-Water (W/W) Emulsions. Curr. Opin. Colloid Interface Sci. 2016, 25, 109-119.

(5) Chao, Y.; Shum, H. C. Emerging Aqueous Two-Phase Systems: From Fundamentals of Interfaces to Biomedical Applications. Chem. Soc. Rev. 2020, 49, 114-142.

(6) Ryden, J.; Albertsson, P.-a. Interfacial Tension of DextranPolyethylene Glycol-Water Two-Phase Systems. J. Colloid Interface Sci. 1971, 37, 219-222.

(7) Broseta, D.; Leibler, L.; Ould Kaddour, L.; Strazielle, C. A Theoretical and Experimental Study of Interfacial Tension of Immiscible Polymer Blends in Solution. J. Chem. Phys. 1987, 87, $7248-7256$.
(8) Ding, P.; Wolf, B.; Frith, W.; Clark, A.; Norton, I.; Pacek, A. Interfacial Tension in Phase-Separated Gelatin/Dextran Aqueous Mixtures. J. Colloid Interface Sci. 2002, 253, 367-376.

(9) Vis, M.; Peters, V. F.; Blokhuis, E. M.; Lekkerkerker, H. N.; Erné, B. H.; Tromp, R. H. Decreased Interfacial Tension of Demixed Aqueous Polymer Solutions Due to Charge. Phys. Rev. Lett. 2015, 115, 078303.

(10) Vis, M.; Peters, V. F.; Tromp, R. H.; Erné, B. H. Donnan Potentials in Aqueous Phase-Separated Polymer Mixtures. Langmuir 2014, 30, 5755-5762.

(11) Tromp, R.; Blokhuis, E. Tension, Rigidity, and Preferential Curvature of Interfaces Between Coexisting Polymer Solutions. Macromolecules 2013, 46, 3639-3647.

(12) Poulin, P.; Weitz, D. Inverted and Multiple Nematic Emulsions. Phys. Rev. E: Stat. Phys., Plasmas, Fluids, Relat. Interdiscip. Top. 1998, 57, 626 .

(13) Rudhardt, D.; Fernandez-Nieves, A.; Link, D.; Weitz, D. Phase Wwitching of Ordered Arrays of Liquid Crystal Emulsions. Appl. Phys. Lett. 2003, 82, 2610-2612.

(14) Loudet, J.; Richard, H.; Sigaud, G.; Poulin, P. Nonaqueous Liquid Crystal Emulsions. Langmuir 2000, 16, 6724-6730.

(15) Poulin, P.; Stark, H.; Lubensky, T.; Weitz, D. Novel Colloidal Interactions in Anisotropic Fluids. Science 1997, 275, 1770-1773.

(16) Mondiot, F.; Wang, X.; de Pablo, J. J.; Abbott, N. L. Liquid Crystal-Based Emulsions for Synthesis of Spherical and Non-Spherical Particles with Chemical Patches. J. Am. Chem. Soc. 2013, 135, 99729975.

(17) Yada, M.; Yamamoto, J.; Yokoyama, H. Spontaneous Formation of Regular Defect Array in Water-In-Cholesteric Liquid Crystal Emulsions. Langmuir 2002, 18, 7436-7440.

(18) Mushenheim, P. C.; Abbott, N. L. Hierarchical Organization in Liquid Crystal-In-Liquid Crystal Emulsions. Soft Matter 2014, 10, 8627-8634.

(19) Chu, G.; Zussman, E. From Chaos to Order: Evaporative Assembly and Collective Behavior in Drying Liquid Crystal Droplets. J. Phys. Chem. Lett. 2018, 9, 4795-4801.

(20) Davidson, P.; Garreau, A.; Livage, J. Nematic Colloidal Suspensions of V2O5 in Water -or Zocher Phases Revisited. Liq. Cryst. 1994, 16, 905-910.

(21) Kim, J. E.; Han, T. H.; Lee, S. H.; Kim, J. Y.; Ahn, C. W.; Yun, J. M.; Kim, S. O. Graphene Oxide Liquid Crystals. Angew. Chem., Int. Ed. 2011, 50, 3043-3047.

(22) Dogic, Z.; Fraden, S. Smectic Phase in a Colloidal Suspension of Semiflexible Virus Particles. Phys. Rev. Lett. 1997, 78, 2417.

(23) Nyström, G.; Arcari, M.; Mezzenga, R. Confinement-Induced Liquid Crystalline Transitions in Amyloid Fibril Cholesteric Tactoids. Nat. Nanotechnol. 2018, 13, 330-336.

(24) Bai, L.; Kämäräinen, T.; Xiang, W.; Majoinen, J.; Seitsonen, J.; Grande, R.; Huan, S.; Liu, L.; Fan, Y.; Rojas, O. J. Chirality from Cryo-Electron Tomograms of Nanocrystals Obtained by Lateral Disassembly and Surface Etching of Never-Dried Chitin. ACS Nano 2020, 14, 6921-6930.

(25) Kaznacheev, A.; Bogdanov, M.; Taraskin, S. The Nature of Prolate Shape of Tactoids in Lyotropic Inorganic Liquid Crystals. J. Exp. Theor. Phys. 2002, 95, 57-63.

(26) Bagnani, M.; Nyström, G.; De Michele, C.; Mezzenga, R. Amyloid Fibrils Length Controls Shape and Structure of Nematic and Cholesteric Tactoids. ACS Nano 2019, 13, 591-600.

(27) Kim, Y.-K.; Shiyanovskii, S. V.; Lavrentovich, O. D. Morphogenesis of Defects and Tactoids during Isotropic-Nematic Phase Transition in Self-Assembled Lyotropic Chromonic Liquid Crystals. J. Phys.: Condens. Matter 2013, 25, 404202.

(28) Simon, K. A.; Sejwal, P.; Gerecht, R. B.; Luk, Y.-Y. Water-InWater Emulsions Stabilized by Non-Amphiphilic Interactions: Polymer-Dispersed Lyotropic Liquid Crystals. Langmuir 2007, 23, $1453-1458$

(29) Habibi, Y.; Lucia, L. A.; Rojas, O. J. Cellulose Nanocrystals: Chemistry, Self-Assembly, and Applications. Chem. Rev. 2010, 110, 3479-3500. 
(30) Ajdary, R.; Tardy, B. L.; Mattos, B. D.; Bai, L.; Rojas, O. J. Plant Nanomaterials and Inspiration from Nature: Water Interactions and Hierarchically Structured Hydrogels. Adv. Mater. 2020, 2001085.

(31) Revol, J.-F.; Bradford, H.; Giasson, J.; Marchessault, R.; Gray, D. Helicoidal Self-Ordering of Cellulose Microfibrils in Aqueous Suspension. Int. J. Biol. Macromol. 1992, 14, 170-172.

(32) Onsager, L. The Effects of Shape on the Interaction of Colloidal Particles. Ann. N. Y. Acad. Sci. 1949, 51, 627-659.

(33) Edgar, C. D.; Gray, D. G. Influence of Dextran on the Phase Behavior of Suspensions of Cellulose Nanocrystals. Macromolecules 2002, 35, 7400-7406.

(34) Chu, G.; Vasilyev, G.; Qu, D.; Deng, S.; Bai, L.; Rojas, O. J.; Zussman, E. Structural Arrest and Phase Transition in Glassy Nanocellulose Colloids. Langmuir 2020, 36, 979-985.

(35) Beck-Candanedo, S.; Viet, D.; Gray, D. G. Induced Phase Separation in Low-Ionic-Strength Cellulose Nanocrystal Suspensions Containing High-Molecular-Weight Blue Dextrans. Langmuir 2006, 22, 8690-8695.

(36) Liu, Q.; Campbell, M. G.; Evans, J. S.; Smalyukh, I. I. Orientationally Ordered Colloidal Co-Dispersions of Gold Nanorods and Cellulose Nanocrystals. Adv. Mater. 2014, 26, 7178-7184.

(37) Wang, P. X.; Hamad, W. Y.; MacLachlan, M. J. Size-Selective Exclusion Effects of Liquid Crystalline Tactoids on Nanoparticles: A Separation Method. Angew. Chem., Int. Ed. 2018, 57, 3360-3365.

(38) Guidetti, G.; Atifi, S.; Vignolini, S.; Hamad, W. Y. Flexible Photonic Cellulose Nanocrystal Films. Adv. Mater. 2016, 28, 1004210047.

(39) Shopsowitz, K. E.; Qi, H.; Hamad, W. Y.; MacLachlan, M. J. Free-Standing Mesoporous Silica Films with Tunable Chiral Nematic Structures. Nature 2010, 468, 422-425.

(40) Giese, M.; Blusch, L. K.; Khan, M. K.; MacLachlan, M. J. Functional Materials from Cellulose-Derived Liquid-Crystal Templates. Angew. Chem., Int. Ed. 2015, 54, 2888-2910.

(41) Chu, G.; Camposeo, A.; Vilensky, R.; Vasilyev, G.; Martin, P.; Pisignano, D.; Zussman, E. Printing Flowers? Custom-Tailored Photonic Cellulose Films with Engineered Surface Topography. Matter 2019, 1, 988-1000.

(42) Chu, G.; Wang, X.; Chen, T.; Xu, W.; Wang, Y.; Song, H.; Xu, Y. Chiral Electronic Transitions of YVO4: Eu3+ Nanoparticles in Cellulose Based Photonic Materials with Circularly Polarized Excitation. J. Mater. Chem. C 2015, 3, 3384-3390.

(43) Chu, G.; Qu, D.; Camposeo, A.; Pisignano, D.; Zussman, E. When Nanocellulose Meets Diffraction Grating: Freestanding Photonic Paper with Programmable Optical Coupling. Mater. Horiz. 2020, 7, 511-519.

(44) Chu, G.; Vilensky, R.; Vasilyev, G.; Deng, S.; Qu, D.; Xu, Y.; Zussman, E. Structural Transition in Liquid Crystal Bubbles Generated from Fluidic Nanocellulose Colloids. Angew. Chem. 2017, 129, 8877-8881.

(45) Chu, G.; Vilensky, R.; Vasilyev, G.; Martin, P.; Zhang, R.; Zussman, E. Structure Evolution and Drying Dynamics in Sliding Cholesteric Cellulose Nanocrystals. J. Phys. Chem. Lett. 2018, 9, $1845-1851$

(46) Hu, Z.; Marway, H. S.; Kasem, H.; Pelton, R.; Cranston, E. D. Dried and Redispersible Cellulose Nanocrystal Pickering Emulsions. ACS Macro Lett. 2016, 5, 185-189.

(47) Peddireddy, K. R.; Nicolai, T.; Benyahia, L.; Capron, I. Stabilization of Water-In-Water Emulsions by Nanorods. ACS Macro Lett. 2016, 5, 283-286.

(48) Cherhal, F.; Cousin, F.; Capron, I. Structural Sescription of the Interface of Pickering Emulsions Stabilized by Cellulose Nanocrystals. Biomacromolecules 2016, 17, 496-502.

(49) Flory, P. J. Statistical Thermodynamics of Mixtures of Rodlike Particles. 5. Mixtures with Random Coils. Macromolecules 1978, 11, $1138-1141$

(50) Bukusoglu, E.; Bedolla Pantoja, M.; Mushenheim, P. C.; Wang, X.; Abbott, N. L. Design of Responsive and Active (Soft) Materials Using Liquid Crystals. Annu. Rev. Chem. Biomol. Eng. 2016, 7, 163196.
(51) Schütz, C.; Agthe, M.; Fall, A. B.; Gordeyeva, K.; Guccini, V.; Salajkova, M.; Plivelic, T. S.; Lagerwall, J. P.; Salazar-Alvarez, G.; Bergström, L. Rod Packing in Chiral Nematic Cellulose Nanocrystal Dispersions Studied by Small-Angle X-Ray Scattering and Laser Diffraction. Langmuir 2015, 31, 6507-6513.

(52) Grinberg, V. Y.; Tolstoguzov, V. Thermodynamic Incompatibility of Proteins and Polysaccharides in Solutions. Food Hydrocolloids 1997, 11, 145-158.

(53) Vis, M.; Opdam, J.; Van't Oor, I. S.; Soligno, G.; Van Roij, R.; Tromp, R. H.; Erné, B. H. Water-In-Water Emulsions Stabilized by Nanoplates. ACS Macro Lett. 2015, 4, 965-968.

(54) Keal, L.; Colosqui, C. E.; Tromp, R. H.; Monteux, C. Colloidal Particle Adsorption at Water-Water Interfaces with Ultralow Interfacial Tension. Phys. Rev. Lett. 2018, 120, 208003.

(55) Tromp, R. H. Water-Water Interfaces. In Structure and Functional Properties of Colloidal Systems; Hidalgo-Alvárez, R., Ed.; Taylor and Francis: Boca Raton, FL, 2016; pp 201-208.

(56) Li, Y.; Suen, J. J.-Y.; Prince, E.; Larin, E. M.; Klinkova, A.; Thérien-Aubin, H.; Zhu, S.; Yang, B.; Helmy, A. S.; Lavrentovich, O. D.; Kumacheva, E. Colloidal Cholesteric Liquid Crystal in Spherical Confinement. Nat. Commun. 2016, 7, 12520.

(57) Thérien-Aubin, H.; Lukach, A.; Pitch, N.; Kumacheva, E. Coassembly of Nanorods and Nanospheres in Suspensions and in Stratified Films. Angew. Chem. 2015, 127, 5710-5714.

(58) Chu, G.; Vasilyev, G.; Vilensky, R.; Boaz, M.; Zhang, R.; Martin, P.; Dahan, N.; Deng, S.; Zussman, E. Controlled Assembly of Nanocellulose-Stabilized Emulsions with Periodic Liquid Crystal-InLiquid Crystal Organization. Langmuir 2018, 34, 13263-13273.

(59) Sarkar, A.; Li, H.; Cray, D.; Boxall, S. Composite Whey ProteinCellulose Nanocrystals at Oil-Water Interface: Towards Delaying Lipid Digestion. Food Hydrocolloids 2018, 77, 436-444.

(60) Mezzenga, R.; Folmer, B. M.; Hughes, E. Design of Double Emulsions by Osmotic Pressure Tailoring. Langmuir 2004, 20, 35743582.

(61) Schneider, C. A.; Rasband, W. S.; Eliceiri, K. W. NIH Image to ImageJ: 25 Years of Image Analysis. Nat. Methods 2012, 9, 671-675.

(62) Bai, L.; Xiang, W.; Huan, S.; Rojas, O. J. Formulation and Stabilization of Concentrated Edible Oil-in-Water Emulsions Based on Electrostatic Complexes of a Food-Grade Cationic Surfactant (Ethyl Lauroyl Arginate) and Cellulose Nanocrystals. Biomacromolecules 2018, 19, 1674-1685. 